\title{
Plasma insulin levels are increased by sertraline in rats under oral glucose overload
}

R. Gomez ${ }^{1}$, J. Huber ${ }^{1}$,

F. Lhullier ${ }^{2}$ and

H.M.T. Barros ${ }^{1}$
${ }^{1}$ Divisão de Farmacologia, Fundação Faculdade Federal de Ciências M édicas de Porto Alegre, Porto Alegre, RS, Brasil

${ }^{2}$ Hospital de Clínicas de Porto Alegre, Porto Alegre, RS, Brasil

\section{Correspondence \\ H.M.T. Barros \\ Divisão de Farmacologia \\ FFFCM PA \\ Rua Sarmento Leite, 245 \\ 90050-170 Porto Alegre, RS \\ Brasil \\ Fax: + 55-51-224-8822 \\ E-mail: helenbar@ fffcmpa.tche.br \\ Research supported by AAPEFATO. \\ R. Gomez was the recipient of a \\ doctoral scholarship from CAPES. \\ H.M.T. Barros is the recipient of \\ a CNPq 1C Productivity Grant.}

Received January 2, 2001

Accepted October 2, 2001

\section{Abstract}

Recognition and control of depression symptoms are important to increase patient compliance with treatment and to improve the quality of life of diabetic patients. Clinical studies indicate that selective serotonin reuptake inhibitors (SSRI) are better antidepressants for diabetic patients than other drugs. However, preclinical trials have demonstrated that not all SSRI reduce plasma glucose levels. In fact, fluoxetine increases and sertraline decreases glycemia in diabetic and non-diabetic rats. In the present study we evaluated plasma insulin levels during fasting and after glucose overload after treatment with sertraline. Adult male Wistar rats were fasted and treated with saline or $30 \mathrm{mg} / \mathrm{kg}$ sertraline and submitted or not to glucose overload $(\mathrm{N}=10)$. Blood was collected and plasma insulin was measured. The mean insulin levels were: fasting group: $25.9 \pm 3.86$, sertraline + fasting group: $31.10 \pm 2.48$, overload group: $34.1 \pm 3.40$, and overload + sertraline group: $43.73 \pm 5.14 \mu \mathrm{U} / \mathrm{ml}$. Insulinemia was significantly increased in the overload + sertraline group. There were no differences between the other groups. No difference in glucose/insulin ratios could be detected between groups. The overload + sertraline group was the only one in which a significant number of individuals exceeded the upper confidence limit of insulin levels. This study demonstrates that sertraline increases glucose-stimulated insulin secretion without any change in peripheral insulin sensitivity.

Depression occurs at much higher rates (15 to $25 \%)$ in diabetic patients than in the general population $(1,2)$, even though depression symptoms are frequently underdiagnosed (3). In diabetic patients, recognition and control of depression symptoms is of the utmost importance to increase compliance with treatment and to improve life quality (4). However, the main concern about antidepressant drug treatment is that several
Key words

- Depression

- Antidepressants

- Diabetes

- Insulinemia commonly used agents alter blood glucose levels of diabetic and non-diabetic patients $(5,6)$ and present important interactions with oral hypoglycemic agents $(7,8)$. More recent clinical studies have indicated that selective serotonin reuptake inhibitors (SSRI) are better antidepressants for diabetic patients because they interfere less with plasma glucose control $(9,10)$. Preclinical trials have shown that some SSRI reduce plasma glucose levels 
$(11,12)$. In fact, sertraline has been shown to decrease fasting and overload glycemia in diabetic and non-diabetic rats (12). On the other hand, within the group of SSRI, sertraline may be the best agent for treatment of depression in diabetic patients (10). The mechanism by which sertraline decreases glycemia might be associated with an increase in plasma insulin concentration after glucose overload, reduction of glucose intestinal absorption, increase in peripheral insulin sensitivity, or intracellular biochemical mechanisms. All of these mechanisms need further evaluation. The purpose of the present study was to evaluate plasma insulin levels during fasting and after glucose overload in rats under treatment with sertraline. Secondarily we determined the glucose/insulin ratio to estimate peripheral insulin sensitivity.

Forty adult male albino Wistar rats raised in the animal house of Fundação Faculdade Federal de Ciências Médicas de Porto Alegre (FFFCMPA) were used in this study. The animals were maintained in groups of five, under controlled room temperature of $22 \pm$ $2^{\circ} \mathrm{C}$ and illumination from 7:00 am to 7:00 pm. Water was available ad libitum. Rat chow (Nutrilab, São Paulo, SP, Brazil) was available up to $18 \mathrm{~h}$ before the beginning of experiments.

A $50 \%$ glucose solution was prepared with glucose (D (+) glucose monohydrate; Merck, Rio de Janeiro, RJ, Brazil) dissolved in distilled water. One milliliter of this solution was administered to each rat by gavage. Sertraline (Zoloft ${ }^{\circledR}$, Pfizer, São Paulo, SP, Brazil), $30 \mathrm{mg} / \mathrm{ml}$, was diluted in distilled water containing $0.05 \%$ Tween 80 . All solutions were prepared immediately before use. Sertraline treatment was administered $i p$ in a volume of $1 \mathrm{ml} / \mathrm{kg}$. The control group received vehicle.

The rats were subdivided randomly into two groups ( $\mathrm{N}=20$ animals each) submitted to determination of fasting or glucose overload levels of insulin. In both groups, 10 rats received $1 \mathrm{ml} / \mathrm{kg}$ saline and the other 10 rats were injected with $30 \mathrm{mg} / \mathrm{kg}$ sertraline, a dose that decreased blood glucose levels in a previous study (12). The rats from the fasting groups were sacrificed by decapitation after $30 \mathrm{~min}$, and blood was collected from the body trunk. Thirty minutes after saline or sertraline administration to the overload groups, $1 \mathrm{ml}$ of a $50 \%$ glucose solution was administered by gavage and the rats were sacrificed for blood collection $30 \mathrm{~min}$ later. Plasma glucose levels were measured with a Glucotrend ${ }^{\circledast}$ device (Boehringer Institute, Mannheim, Germany). Plasma insulin was determined by radioimmunoassay using a polyclonal antibody (Coat-A-Count ${ }^{\circledR}$, Diagnostic Products Corp., Los Angeles, CA, USA). The Ethics Committee for Experimental Procedures of FFFCMPA approved this experimental protocol.

The insulin levels and the glucose/insulin ratios (13) of the four groups were analyzed using the Sigma Stat 2.0 software (Jandel ${ }^{\circledR}$, Chicago, IL, USA). One-way ANOVA was used to compare insulin levels or glucose/insulin ratios of the four treatment groups, followed by the Student Newman-Keuls test for post hoc comparisons. The $95 \%$ confidence interval was calculated to estimate the standard levels of plasma insulin of fasting rats. The Fisher exact test was applied to compare the number of individuals in the treatment groups whose fasting insulin levels exceeded the upper limit. Group differences were accepted when $\mathrm{P}<0.05$. Data are reported as mean $\pm \mathrm{SEM}$.

Mean insulinemia was $25.9 \pm 3.86 \mu \mathrm{U} /$ $\mathrm{ml}$ in the fasting group, $31.10 \pm 2.48 \mu \mathrm{U} / \mathrm{ml}$ in the sertraline + fasting group, $34.1 \pm 3.40$ $\mu \mathrm{U} / \mathrm{ml}$ in the overload group, and $43.73 \pm$ $5.14 \mu \mathrm{U} / \mathrm{ml}$ in the overload + sertraline group, with significant differences between groups $\left(\mathrm{F}_{(3,37)}=3.700 ; \mathrm{P}=0.021\right)$. The group treated with sertraline presented a significant increase in insulinemia after the glucose overload (Figure 1). There was no difference between the other groups. 
The upper $95 \%$ confidence limit of fasting insulinemia of rats was $34.65 \mu \mathrm{U} / \mathrm{ml}$. After glucose overload, five of the ten animals presented insulin levels above the upper confidence limit $(\mathrm{P}>0.05)$. When $30 \mathrm{mg} /$ $\mathrm{kg}$ sertraline was administered to fasting animals, only three of the ten animals exceeded the confidence limit $(\mathrm{P}>0.05)$. The only group in which a significant number of individuals showed insulin levels exceeding the confidence limit ( 8 of 10 animals) was the one treated with sertraline and submitted to glucose overload $(\mathrm{P}>0.05)$.

To estimate and compare insulin sensitivity, the glucose/insulin ratio was calculated for each group and found to be $2.71 \pm$ $0.9 \mathrm{mg} \mathrm{dl}^{-1} \mu \mathrm{U} / \mathrm{ml}^{-1}$ for the fasting group and $2.84 \pm 1.0 \mathrm{mg} \mathrm{dl}^{-1} \mu \mathrm{U} / \mathrm{ml}^{-1}$ for the glucose overload group. After treatment with $30 \mathrm{mg} /$ $\mathrm{kg}$ sertraline, the glucose/insulin ratio was $1.86 \pm 1.6 \mathrm{mg} \mathrm{dl}^{-1} \mu \mathrm{U} / \mathrm{ml}^{-1}$ in the fasting group and $1.26 \pm 3.1 \mathrm{mg} \mathrm{dl}^{-1} \mu \mathrm{U} / \mathrm{ml}^{-1}$ in the overload group. No difference in glucose/ insulin ratios was detected between groups $(\mathrm{P}>0.05)$.

The results of the present experiment demonstrate that acute dosing with $30 \mathrm{mg} / \mathrm{kg}$ sertraline does not change plasma insulin levels of fasting animals but increases glucose-stimulated insulin secretion.

The administration of sertraline reduces glycemia in fasting diabetic mice (14) and decreases fasting and glucose overload glycemia in diabetic and non-diabetic rats (12). However, in the present study, insulinemia was increased by sertraline treatment only after glucose overload. In fact, in fasting diabetic mice, sertraline also does not change plasma insulin concentrations (12). Thus, it is possible that sertraline presents this interesting hypoglycemia reducing effect by more than one mechanism. This study demonstrated that sertraline increases glucose-stimulated insulin secretion without any change in peripheral insulin sensitivity. Additional studies will be necessary to evaluate if reduction of intestinal glucose absorp- tion or increase in intracellular cascades for glucose utilization are the mechanisms involved.

In a previous study we showed that sertraline decreases glucose overload glycemia. In the present study we confirm this information and extend it by showing that a possible mechanism underlying this effect is an increase in insulin levels. No pharmacokinetic interaction between sertraline and insulin has been reported even though large doses of sertraline associated with sulfonylurea potentiate hypoglycemia by enzymatic competition for the cytochrome P-450 metabolic system (8).

Antidepressant drugs such as imipramine and monoamine oxidase inhibitors are usually avoided by diabetic patients due to the risk of hyperglycemia $(5,6)$ and to the possibility of disrupting the antidiabetic effects of oral hypoglycemic agents and insulin $(7,8)$. Thus, treatment of depressive diabetic individuals with these agents should be prescribed with caution. The present data obtained with rats might be used as preclinical

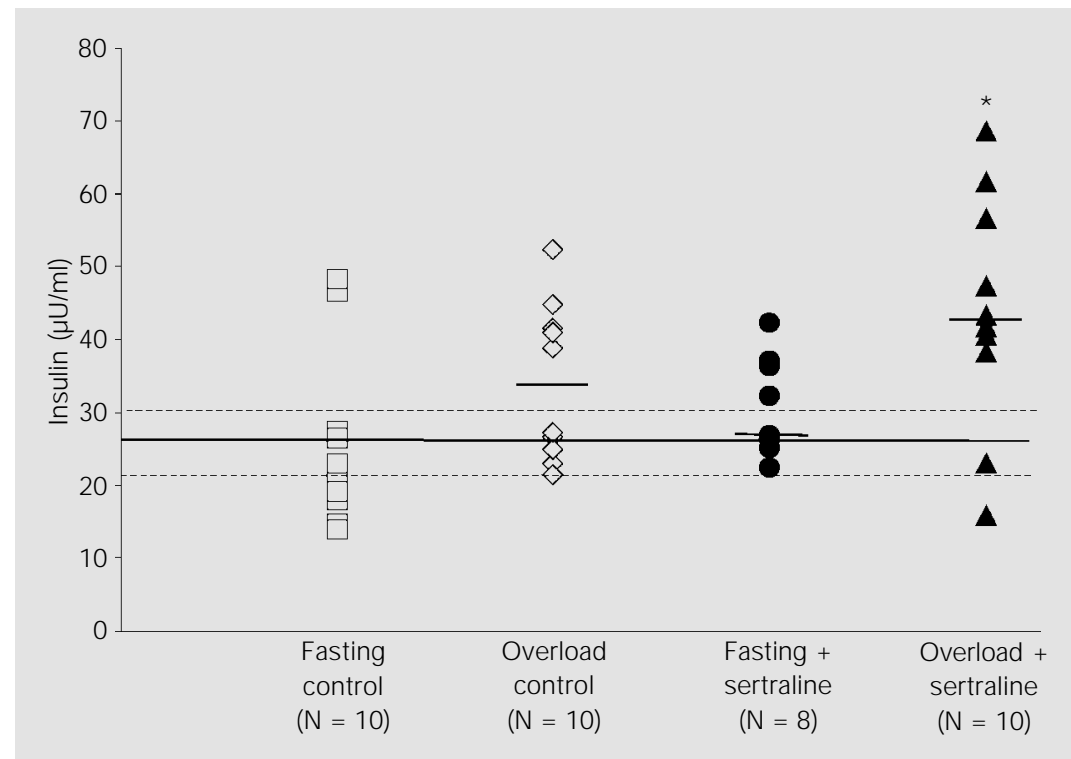

Figure 1. Individual insulinemia of rats determined after fasting and after $30 \mathrm{~min}$ of oral glucose overload, with or without $30 \mathrm{mg} / \mathrm{kg}$ sertraline treatment. The bold line indicates the mean plasma insulin level of fasting animals treated with saline, and the dotted lines indicate the corresponding upper and lower $95 \%$ confidence limits. $* \mathrm{P}<0.05$ compared to the other groups (Student-Newman-Keuls test). 
evidence to justify future clinical studies with sertraline as an antidepressant for diabetic patients.

Overall, treatment of depression in diabetic patients increases weight loss and compliance with treatment with oral hypoglycemic agents, resulting in fewer hypoglycemic crises and delaying long-term diabetes-related complications $(4,15)$. Among the antidepressant drugs, the SSRI have proved to be a good choice for depressed diabetic patients $(1,16)$. In diabetic patients, fluoxetine improves glycemic control, increases weight loss and may be followed by decreased doses of insulin or oral hypoglycemic agents (16). However, it is possible that its beneficial effects in reducing the doses of hypoglycemic agents is due to increased sensitivity of hepatic and peripheral insulin receptors, as demonstrated in obese diabetic individuals with peripheral insulin resistance (15), and to an increase in glycogen synthetase activity with no changes in insulinemia (4). Nevertheless it is possible that sertraline is a better agent to be administered to human diabetics compared to fluoxetine, since sertraline induces only minor antimuscarinic effects, such as dry mouth, constipation and cognitive changes; thus, it is safe for diabetic patients (10). Our results support the view that sertraline is an effective SSRI agent for depression in diabetic patients. However, they also point to the necessity of dose adjustment of hypoglycemic agents since sertraline increases glucose-stimulated insulin secretion in rats.

\section{References}

1. Goodnick PJ , Henry J H \& Buki VM (1995). Treatment of depression in patients with diabetes mellitus. J oumal of Clinical Psychiatry, 56: 128-136.

2. Lustman PJ , Clouse RE \& Freedland KE (1998). Management of major depression in adults with diabetes: implications of recent clinical trials. Seminars in Clinical Neuropsychiatry, 3: 102-114.

3. Gavard J A, Lustman PJ \& Clouse RE (1993). Prevalence of depression in adults with diabetes: an epidemiological evaluation. Diabetes Care, 16: 1167-1178.

4. Breum L, Bjerre U, Bak J F, J acobsen S \& Astrup A (1995). Long-term effects of fluoxetine on glycemic control in obese patients with non-insulin-dependent diabetes mellitus or glucose intolerance: influence on muscle glycogen synthase and insulin receptor kinase activity. Metabolism, 44: 1570-1576.

5. Kaplan SM, Mass J W, Pixley J M \& Ross D (1960). Use of imipramine in diabetes. Effects on glycosuria and blood sugar levels. J ournal of the American Medical Association, 174: 511-517.

6. Lundquist I, Ekholm R \& Ericson LE
(1971). Monoamines in the pancreatic islets of the mouse. 5-Hydroxytryptamine as an intracellular modifier of insulin secretion, and the hypoglycaemic action of monoamine oxidase inhibitors. Diabetologia, 7: 414-422.

7. Cooper AJ \& Ashcroft G (1963). Potentiation of insulin hypoglycaemia by MAOI antidepressant drugs. Lancet, 1: 407-409.

8. Takhar J \& Williamson P (1999). Hypoglycaemia associated with high doses of sertraline and sulfonylurea compound in a non-insulin-dependent diabetes mellitus patient. Canadian J ournal of Pharmacology, 6: 12-14.

9. Warnock J K \& Mutzig EM (1998). Diabetes mellitus and major depression: considerations for treatment of native Americans. J ournal of Oklahoma State Medical Association, 91: 488-493.

10. Goodnick PJ, Kumar A, Henry J H, Buki VM \& Goldberg RB (1997). Sertraline in coexisting major depression and diabetes mellitus. Psychopharmacology Bulletin, 33: 261-264.

11. Furlan BL \& Wilson A (1980). Further studies on the effects of 5-hydroxytryptophan on plasma glucose and insulin in the mouse. Diabetologia, 19: 386-390.

12. Gomez R, Huber J, Tombini G \& Barros HMT (2001). Acute effect of different antidepressants on glycemia in diabetic and non-diabetic rats. Brazilian J ournal of Medical and Biological Research, 34: 5764.

13. Caro J F (1991). Clinical review 26: Insulin resistance in obese and nonobese man. J ournal of Clinical Endocrinology and Metabolism, 73: 691-695.

14. Erenmemisoglu A, Ozdogan UK, Sarayemen R \& Tutus A (1999). Effect of some antidepressants on glycemia and insulin levels of normoglycaemic and alloxan-induced hyperglycaemic mice. J oumal of Pharmacy and Pharmacology, 51: 741743.

15. Poter van Loon BJ , Radder J K \& Frolich M (1992). Fluoxetine increases insulin action in obese nondiabetic and in obese noninsulin-dependent diabetic individuals. Intemational J ournal of Obesity, 16: 79-85.

16. Wise SD (1992). Clinical studies with fluoxetine in obesity. American J ournal of Clinical Nutrition, 55: 181S-184S. 